\title{
Erratum to: Fatty acid composition in fetal, neonatal, and cultured cardiomyocytes in rats
}

\author{
Toru Karimata $^{1}$ - Daisuke Sato ${ }^{1}$ Daiki Seya ${ }^{2}$ - Daichi Sato ${ }^{1} \cdot$ Takashi Wakatsuki $^{1}$. \\ Zhonggang Feng ${ }^{2} \cdot$ Atsuyoshi Nishina ${ }^{3} \cdot$ Masataka Kusunoki $^{4} \cdot$ Takao Nakamura $^{1}$
}

Published online: 4 February 2016

(C) The Society for In Vitro Biology 2016

\section{Erratum to: In Vitro Cell. Dev. Biol.-Animal}

DOI: 10.1007/s11626-013-9668-3

\section{Published Vol. 49 No.10 pp. 798-804}

In fatty acid analyses of our paper, we found that we had mistaken chromatographic peak of docosapentaenoic acid (C22:5n-3) for that of nervonic acid (C24:1n-9).

Consequently, first, C24:1n-9 was not detected in any data. Therefore, C24:1n-9 data needs to be omitted in Figure 2 in the paper, and in the sentence "Other MUFA contents in the Neo group were generally lower than those in the Fet and $\mathrm{Cul}$

The online version of the original article can be found at http:dx.doi.org/ 10.1007/s11626-013-9668-3.

Daisuke Sato

d_sato@yz.yamagata-u.ac.jp

1 Department of Biomedical Information Engineering, Graduate School of Medical Science, Yamagata University, 2-2-2, Iida-nishi, Yamagata 990-9585, Japan

2 Department of Bio-Systems Engineering, Graduate School of Science and Engineering, Yamagata University, 4-3-16, Johnan, Yonezawa 992-8510, Japan

3 Department of Materials and Applied Chemistry, College of Science and Technology, Nihon University, 1-8-14, Kandasurugadai, Chiyoda-ku, Tokyo 101-8308, Japan

4 Department of Internal Medicine, Medical Clinic, Aichi Medical University, 2-12-1, Higashisakura, Higashi-ku, Nagoya 461-0005, Japan

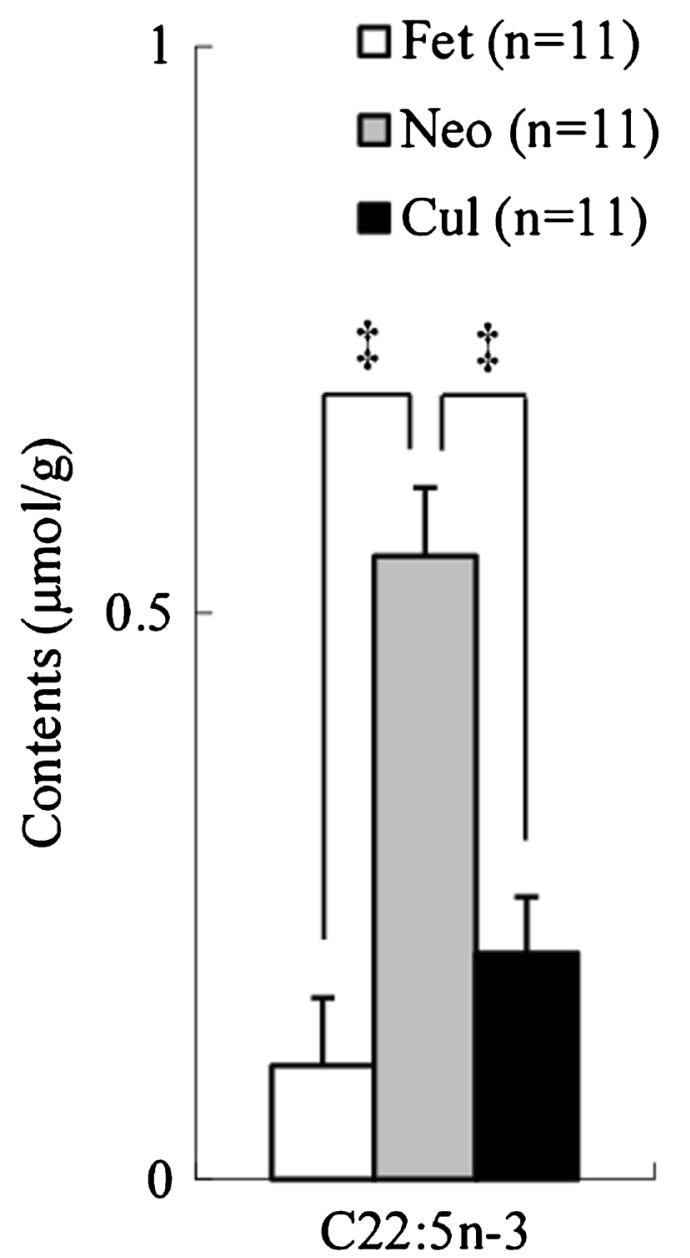

Figure 3 Corrected C22:5n-3 content. Data is expressed as mean $\pm \mathrm{SE}$. Significant difference among the groups were detected by non-parametric Steel-Dwass test. $₫ p<0.01$. 
groups, except for nervonic acid (C24:1n-9) where the content in the Neo group was the highest $(p<0.01)$ " (3rd sentence of 4th paragraph in the Results section of the paper), the phrase starting with "except" needs to be deleted.

Second, C22:5n-3 content in the Neo group became significantly higher than those in the Fet and Cul groups $(p<0.01$, respectively) as shown in Figure 3, and the sentence "Some PUFAs in the Fet group were higher $(\mathrm{C} 22: 5 n-3, p<0.05)$ or lower $(\mathrm{C} 18: 3 n-3, p<0.05$; $\mathrm{C} 22: 6 n-3, p<0.05)$ than those in the Neo group, and higher than those in the $\mathrm{Cul}$ group (C20:4n-6 and C22:4n-6, $p<0.01$ )" (last paragraph in the Results section of the paper) needs to be substituted for "Some PUFAs in the Fet group were lower (C18:3n-3 and C22:6n-3, $p<0.05$; C22:5n-3, $p<0.01)$ than those in the Neo group, and higher than those in the Cul group (C20:4n-6 and C22:4n-6, $p<0.01)^{\prime \prime}$.

The revision shown above does not influence our conclusion. 